\title{
A CHARACTERIZATION OF GROUPS WITH ISOMORPHIC SUBGROUPS
}

\author{
DONALD W. MILLER
}

\begin{abstract}
The concept of normalizer is generalized to derive a characterization of groups $G$ which contain a proper subgroup isomorphic to $G$.
\end{abstract}

The problem of what groups have proper isomorphic subgroups has attracted the attention of a number of authors. Baer [1] obtained a characterization of groups without proper isomorphic subgroups, while Beaumont [2], Kaplansky [4] and Clay [3] identified a number of classes of groups with proper isomorphic subgroups as well as several classes of groups without such subgroups. It is the purpose of this note to give an explicit characterization of groups with proper isomorphic subgroups. ${ }^{1}$

Let $H$ be a subgroup of the group $G$, with normalizer $N=N_{G}(H)$, where $N=\{x \in G \mid x H=H x\}$. Define the left normalizer, $L=L_{G}(H)$, of $H$ in $G$ by $L=\{x \in G \mid x H \subseteq H x\}$ and the right normalizer, $R=R_{G}(H)$, of $H$ in $G$ by $R=\{x \in G \mid H x \subseteq x H\}$. The following observations are immediate consequences of these definitions.

Lemma. Let $H$ be a subgroup of a group $G$. Then:

(i) $L \cap R=N$;

(ii) $R=L^{-1}=\left\{x^{-1} \mid x \in L\right\}$;

(iii) either $L=R=N$, or $L, R$ and $N$ are pairwise distinct.

The criterion referred to in the title can now be established.

THEOREM. A group $H$ has an isomorphic proper subgroup if and only if $H$ is imieddable in a group $G$ with the property that the left and right normalizers of $H$ in $G$ are distinct.

Proof. Suppose that $H$ is imbeddable in a group $G$ such that $L=$ $L_{G}(H) \neq R=R_{G}(H)$. Then $L \supset N=N_{G}(H)$ so, selecting $a$ in $L \backslash N$ (the complement of $N$ in $L$ ), we have $a H \subset H a$. Thus, $a H a^{-1}$ is a proper subgroup of $H$ which is isomorphic to $H$.

Received by the editors July $12,1971$.

AMS 1970 subject classifications. Primary 20E15.

Key words and phrases. Isomorphic subgroup, normalizer, holomorph.

${ }^{1}$ The generalizations of the normalizer of a subgroup on which this characterization is based were suggested to the writer by Thomas Shores.

(c) American Mathematical Society 1972 
Conversely, let $H_{1}$ be a group which contains a proper isomorphic subgroup $H_{0}$, with $\varphi_{0}$ an isomorphism of $H_{0}$ onto $H_{1}$. Then

$$
H_{0} \varphi_{0}=H_{1} \supset H_{0}
$$

so there exists a proper subgroup $H_{-1}=H_{0} \varphi_{0}^{-1}$ of $H_{0}$ such that $H_{-1} \varphi_{0}=H_{0}$. Consequently $\varphi_{-1}=q_{0} \mid H_{-1}$ (the restriction of $\varphi_{0}$ to $H_{-1}$ ) is an isomorphism of $H_{-1}$ onto $H_{0}$. Iteration of this process leads to a strictly decreasing chain of subgroups $H_{1} \supset H_{0} \supset H_{-1} \supset \cdots$ of $H_{1}$, together with a sequence of surjective isomorphisms $\varphi_{i}: H_{i} \rightarrow H_{i+1}, i=0,-1,-2, \cdots$, such that

$$
\varphi_{i} \mid H_{i-1}=\varphi_{i-1}, \quad i=0,-1,-2, \cdots .
$$

Let $A_{1}$ be a set disjoint from $H_{1}$ such that $\left|A_{1}\right|=\left|H_{1} \backslash H_{0}\right|$, and let $\beta$ be a bijection from $H_{1} \backslash H_{0}$ to $A_{1}$. Define a bijection $\varphi_{1}$ from $H_{1}$ to $H_{2}=H_{1} \cup A_{1}$ by

$$
\varphi_{1}\left|H_{0}=\varphi_{0}, \quad \varphi_{1}\right|\left(H_{1} \backslash H_{0}\right)=\beta .
$$

Extend the definition of multiplication from $H_{1}$ to $H_{2}$ by stipulating that

$$
x y=\left(\left(x q_{1}^{-1}\right)\left(y q_{1}^{-1}\right)\right) p_{1}, \quad \text { all } x, y \in H_{2},
$$

(which is clearly consistent with the existing multiplication in $H_{1}$ ). Then for all $x, y \in H_{1}$,

$$
\left(x q_{1}\right)\left(y \varphi_{1}\right)=\left(\left(x \varphi_{1} \varphi_{1}^{-1}\right)\left(y \varphi_{1} \varphi_{1}^{-1}\right)\right) \varphi_{1}=(x y) \varphi_{1},
$$

so $\varphi_{1}$ is an isomorphism of $H_{1}$ onto $H_{2}$.

Repetition of this procedure yields a strictly increasing chain of groups $H_{1} \subset H_{2} \subset H_{3} \subset \cdots$, together with a sequence of isomorphisms $\varphi_{i}$ : $H_{i} \rightarrow H_{i+1}, i=1,2,3, \cdots$, such that

$$
\varphi_{i} \mid H_{i-1}=\varphi_{i-1}, \quad i=1,2,3, \cdots .
$$

Now let $K$ be the group given by $K=\bigcup_{i=-\infty}^{\infty} H_{i}$ and define the transformation $\varphi$ of $K$ by $\varphi: x \mapsto x \varphi_{i}$, all $x \in H_{i}$; by (1) and (2), $\varphi$ is well defined. It follows that $\varphi$ is an automorphism of $K$. Thus, $\varphi$ is induced by an inner automorphism, say $T(\alpha)$, of the holomorph $G$ of $K$. Consequently,

$$
\alpha^{-1} H_{1} \alpha=H_{1} T(\alpha)=H_{1} \varphi=H_{1} p_{1}=H_{2} \supset H_{1},
$$

so $H_{1} \propto \supset \alpha H_{1}$. Therefore $\alpha \in L_{G}\left(H_{1}\right) \backslash N_{G}\left(H_{1}\right)$, so, by the lemma, $L_{G}\left(H_{1}\right) \neq$ $R_{G}\left(H_{1}\right)$.

\section{BIBLIOGRAPHY}

1. R. Baer, Groups without proper isomorphic quotient groups, Bull. Amer. Math. Soc. 50 (1944), 267-278. MR 5, 228.

2. R. A. Beaumont, Groups with isomorphic proper subgroups, Bull. Amer. Math. Soc. 51 (1945), 381-387. MR 7, 5. 
3. J. R. Clay, The punctured plane is isomorphic to the unit circle, J. Number Theory 1 (1969), 500-501. MR 40 \#227.

4. 1. Kaplansky, A note on groups without isomorphic subgroups, Bull. Amer. Math. Soc. 51 (1945), 529-530. MR 7. 2.

Department of Mathematics, University of Nebraska, Lincoln, Nebraska 68508 